\title{
Three Functionally Distinct Adhesions in Filopodia: Shaft Adhesions Control Lamellar Extension
}

\author{
Michael B. Steketee ${ }^{1}$ and Kathryn W. Tosney ${ }^{1,2}$ \\ ${ }^{1}$ Neuroscience Program and 2Department of Molecular, Cellular, and Developmental Biology, The University of Michigan, \\ Ann Arbor, Michigan 48109
}

In this study, adhesions on individual filopodial shafts were shown to control veil (lamellar) advance and to be modulated by guidance cues. Adhesions were detected in individual filopodia of sensory growth cones using optical recordings, adhesion markers, and electron microscopy. Veils readily advanced along filopodia lacking shaft adhesions but rarely advanced along filopodia displaying shaft adhesions. Experiments altering adhesion showed that this relationship is not caused by veils removing adhesions as they advanced. Reducing adhesion with antibodies decreased the proportion of filopodia with shaft adhesions and coordinately increased veil advance. Moreover, the inhibitory relationship was maintained: veils still failed to advance on individual filopodia that retained shaft adhesions. These results support the idea that shaft adhesions inhibit veil advance. Of particular interest, guidance cues can act by altering shaft adhesions. When a cellular cue was contacted by a filopodial tip, veil extension and shaft adhesions altered in concert. Contact with a Schwann cell induced veil advance and inhibited shaft adhesions. In contrast, contact with a posterior sclerotome cell prohibited veil advance and promoted shaft adhesions. These results show that veil advance is controlled by shaft adhesions and that guidance signal cascades can alter veil advance by altering these adhesions. Shaft adhesions thus differ functionally from two other adhesions identified on individual filopodia. Tip adhesions suffice to signal. Basal adhesions do not influence veil advance but are critical to filopodial initiation and dynamics. Individual growth cone filopodia thus develop three functionally distinct adhesions that are vital for both motility and navigation.

Key words: adhesion; filopodia; growth cone; guidance; lamella; Schwann; sclerotome
The growth cone at the tip of the neurite navigates by probing the environment with its filopodia (Fig. 1). Filopodia are essential for navigation. When they are suppressed, the growth cone can advance but cannot navigate (Bentley and Toroian-Raymond, 1986). Navigation can be mediated by filopodial adhesion. For instance, a growth cone "chooses" between substrates when its filopodia adhere to alternative substrates (Letourneau, 1975). Choice is not attributable to the strength of adhesion. Instead, adhesions modulate second messenger systems that alter cytoskeletal dynamics and thereby direct growth cones (for review, see Gallo and Letourneau, 1999; Isbister and O'Connor, 1999). Indeed, adhesions confined to the tip of a single filopodium can suffice to reorient a growth cone (Hammerback and Letourneau, 1986; O’Connor et al., 1990; Chien et al., 1993; Oakley and Tosney, 1993). Filopodial adhesions are manifestly important to guidance.

Filopodia support and control lamellar extensions termed "veils," which are crucial for growth cone directionality (Goldberg and Burmeister, 1986). Veils may retract, thereby preventing advance; veils may engorge with cytoplasm, thereby promoting advance; selective veil advance or pruning on one side of a growth cone can cause turning (Goldberg and Burmeister, 1986). Veil fate is finely tuned by filopodial adhesion to axonal guidance cues.

Received March 12, 2002; revised June 18, 2002; accepted June 21, 2002.

This work was supported by National Institutes of Health Grant NS21308. We thank Ken Balazovich for critical comments and ultrastructural contributions.

Correspondence should be addressed to Kathryn W. Tosney, Department of Molecular, Cellular, and Developmental Biology, 3103 Natural Science Building, 830 North University, The University of Michigan, Ann Arbor, MI 48109-1048. E-mail: ktosney@umich.edu.

Copyright (C) 2002 Society for Neuroscience $\quad 0270-6474 / 02 / 228071-13 \$ 15.00 / 0$
Veil extension or retraction can be selectively induced when filopodial tips adhere to cues (Burmeister and Goldberg, 1988; O’Connor et al., 1990; Oakley and Tosney, 1993; Polinsky et al., 2000). Adhesion even by a single filopodial tip can suffice to alter veil dynamics, which alters the direction of a growth cone (Bastmeyer and Stuermer, 1993; Oakley and Tosney, 1993; Fan and Raper, 1995; Steketee and Tosney, 1999; Polinsky et al., 2000). Understanding how filopodial adhesions regulate veil activities is thus essential to understanding guidance.

This study elucidated the relationship between veil advance and filopodial adhesions. Veil advance is a process operationally separable from veil initiation. Veil advance is the forward progression of the veil, whereas initiation is the initial emergence of the veil. Veil advance can be inhibited or stimulated by guidance cues that leave veil initiation unaltered (Oakley and Tosney, 1993; Polinsky et al., 2000) and thus is an aspect of veil dynamics that is directly relevant to guidance.

By focusing on adhesions along individual filopodia, this study detected a functionally distinct adhesion class, "shaft adhesions," that lie along shafts of individual filopodia and control veil advance. Shaft adhesions are systematically altered when filopodial tips adhere to physiologically relevant guidance cues. In contrast, veil advance is not affected by a specialized adhesion at the filopodial base. These "basal adhesions" form at the prospective base of each filopodium before it emerges, play a specialized role during filopodial emergence, remain at filopodial bases throughout the lifetime of filopodia, and are associated with a specialized organelle, the "focal ring" (Steketee et al., 2001).

Filopodia thus display three adhesions that have distinctive functions (Fig. 1). Shaft adhesions control veil advance. Tip 


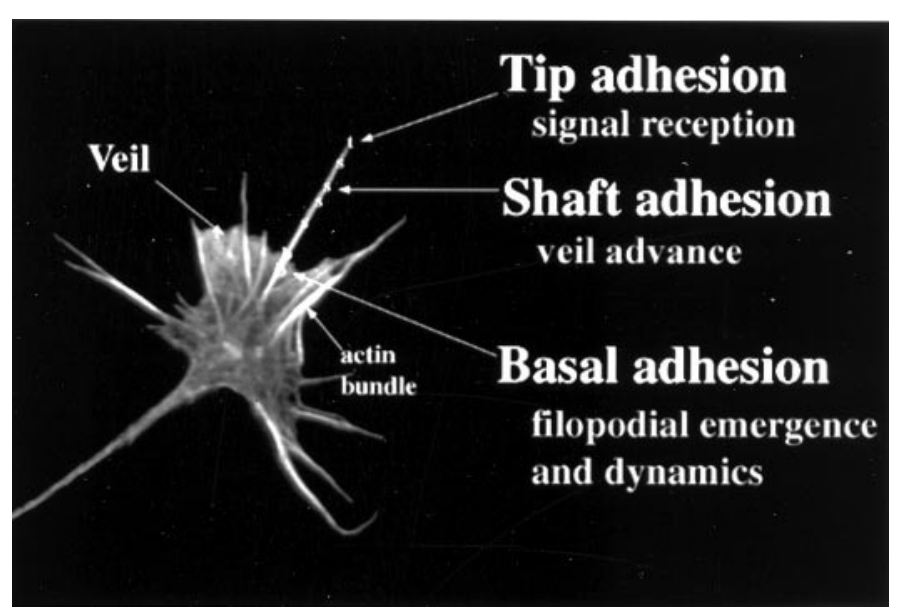

Figure 1. Three functionally distinct adhesions on individual filopodia. The growth cone, the leading tip of the axon, has a central region that consolidates proximally to form the axon. Of primary interest, it has a flat, optically accessible peripheral region that extends veils and filopodia. Filopodia contain a core of actin filaments, whereas veils are actively advancing lamellas containing dendritic actin arrays. Individual filopodia can display three functionally distinct adhesions. Tip adhesions to guidance cues activate signal cascades and suffice to alter discrete aspects of motility, such as veil advance. Basal adhesions lie at filopodial bases, function in filopodial emergence and dynamics, and associate with focal rings. Shaft adhesions lie along distal shafts, lack an association with focal rings, and control veil advance (present study).

adhesions initiate signal cascades that can modulate shaft adhesions and thereby control veil advance. Basal adhesions control filopodial dynamics. Different filopodial adhesions play distinctive and vital roles in growth cone motility and navigation.

\section{MATERIALS AND METHODS}

Cell culture. Sensory neurons were cultured as described by Steketee and Tosney (1999). Briefly, dorsal root ganglia were removed from chick embryos (stage 24-25) (Hamburger and Hamilton, 1951), rinsed in neuron media (NM) composed of Ham's F12 (Invitrogen, Grand Island, NY), and supplemented with $10 \%$ heat-inactivated horse serum, nerve growth factor $(50 \mathrm{ng} / \mathrm{ml})$, HEPES $(10 \mathrm{~mm})$, antibiotics, and hormone additives (Bottenstein et al., 1980). Ganglia were dissociated by pipetting and were equilibrated in a dish of NM for $\geq 3 \mathrm{hr}$. Small explants (10-20 cells) were then plated in $100 \mu \mathrm{l}$ wells on glass coverslips coated with polyornithine (overnight) and laminin $(5 \mathrm{hr})$ and maintained at $37^{\circ} \mathrm{C}, 5 \%$ $\mathrm{CO}_{2}$ until recording $(3-6 \mathrm{hr})$. To assess cellular interactions, neuronal explants were added to either posterior sclerotome or Schwann cell cultures, and 10 interactions with each cell type were analyzed in detail. Posterior sclerotome cells were isolated as described previously (Oakley and Tosney, 1993). Briefly, small explants were selectively aspirated from posterior somites (stage 17-18) (Hamburger and Hamilton, 1951), washed in NM, and allowed to spread overnight on laminin-coated coverslips. To isolate Schwann cells, dorsal roots were removed from stage 24-25 embryos and cultured overnight as described above. Culture purities were verified both morphologically and with antibodies HNK-1 (Kate Barald, University of Michigan) and 1E8 (Erick Frank, University of Pittsburgh, Pittsburgh, PA).

Optical recording. Cells were recorded as described by Oakley and Tosney (1993). During recording, cultures were overlaid with mineral oil $(2 \mathrm{ml})$ and maintained at $37^{\circ} \mathrm{C}$. Interactions were viewed with phasecontrast optics (Nikon Plan Apo 60×/1.40 DM objective; Nikon, Melville, NY) and recorded with a Hamamatsu cooled CCD camera (model C5985; Hamamatsu Photonics, Oak Brook, IL) under control of the Metamorph program (Universal Imaging, West Chester, PA). Images were recorded at 15 frames/min and stored on optical disk (model TQ3038f; Panasonic, Secaucus, NY). In untreated cultures, growth cones were recorded for $\geq 15$ min before fixation $(n=33)$. During cellular interactions $(n=20)$ and antibody treatments $(n=12)$, recorded precontract and postcontact periods were each $\geq 10 \mathrm{~min}$. For presentation, selected images were captured using Metamorph; contrast enhanced, cropped, and combined using Adobe PhotoShop (Adobe Systems, San Jose, CA); and labeled using Adobe Illustrator.

Fixation. To preserve adhesion-stabilized vinculin and phosphotyrosine (Pty), cultures were fixed and extracted simultaneously with $1 \%$ paraformaldehyde, $0.4 \mathrm{M}$ sucrose, and $0.5 \%$ Triton X-100 in Kreb's buffer (Meiri and Burdick, 1991) while recording as described by Steketee et al. (2001). Fixative was applied by layering $2 \mathrm{ml}$ on top of the mineral oil. The solution dropped through the oil, fixing the cultures rapidly and gently. Cultures were fixed for $10 \mathrm{~min}$ at room temperature and then washed three times with each of the following: PBS, $0.5 \mathrm{~m}$ glycine in PBS, and block (1\% BSA in PBS). To prevent differences in preservation among different experiments, a large stock of fixative was aliquoted, frozen, and used in all of the cultures.

Immunocytochemistry. Vinculin and Pty were localized as described by Steketee et al. (2001). Fixed cultures were incubated with either an anti-vinculin monoclonal antibody (Sigma, St. Louis, MO) or an anti-Pty monoclonal antibody (Pt-66; Sigma), diluted in block (1:100), either overnight at $4^{\circ} \mathrm{C}$ or for $2 \mathrm{hr}$ at $37^{\circ} \mathrm{C}$. Cultures were then washed with PBS and block, incubated with an anti-mouse $\operatorname{IgG}$ secondary antibody conjugated to rhodamine (Jackson ImmunoResearch, West Grove, PA) for 30 min, washed with block three times, and mounted in Prolong (Molecular Probes, Eugene, OR) to minimize photobleaching. Cultures were viewed with conventional epifluorescence (Nikon), and images were recorded on optical disk.

Electron microscopy. Gold, 50 mesh electron microscope grids were coated with $0.6 \%$ formvar and lifted onto acid-washed coverslips. Coverslips were affixed to the bottoms of tissue culture dishes, and the assembly was coated with polyornithine and laminin as described above. Guiding cells and small dorsal root ganglion explants were plated onto these culture dishes in NM, and motile activities were recorded onto optical disks as described above. Fixative was added before recording ended. Cells were fixed with $2 \%$ glutaraldehyde in PHEM-N buffer (in mM): 60 PIPES, 25 HEPES, pH 6.9, 10 EGTA, $2 \mathrm{MgCl}_{2}$, and $7.4 \mathrm{NaCl}$, $350 \mathrm{mOsM}$, for $30 \mathrm{~min}$ at room temperature. Fixed cells were washed in PHEM-N and then in water, postfixed with aqueous $0.1 \%$ osmium tetroxide for $5 \mathrm{~min}$, dehydrated through a graded series of ethanol solutions, stained with ethanolic uranyl acetate, and further dehydrated through hexamethyldisilazane (Electron Microscopy Sciences, Fort Washington, PA). Growth cones were observed intact on the grids using a Phillips (FEI Corporation, Hillsboro, OR) CM10 electron microscope operating at $80 \mathrm{kV}$ and photographed onto Kodak 4489 electron image film (Eastman Kodak, Rochester, NY). To test whether shaft adhesions could be discriminated with electron microscopy (EM), we compared stereo images of filopodia that had been moving $(n=20)$ with those of filopodia that were static $(n=12)$ during the last minute before fixation. Substrate specializations characterized static but not moving filopodia. Stereo images of filopodia were then analyzed for substrate specializations in recorded growth cones (50 filopodia supporting veils that were advancing, 28 filopodia supporting veils that had stopped, 14 filopodia from four growth cones contacting Schwann cells, and 12 filopodia from five growth cones contacting posterior sclerotome).

Analysis. Veils were defined as thin sheets of lamella that advanced $\geq 2$ $\mu \mathrm{m}$ from the growth cone margin. The sampling frequency (1 frame every $4 \mathrm{sec}$ ) was selected to be shorter than required for detecting veils. Veils advance at a strikingly consistent rate that does not change significantly even after contact with guidance cues (Steketee and Tosney, 1999). Veils advance at $7 \pm 1 \mu \mathrm{m} / \mathrm{min}(\sim 0.12 \mu \mathrm{m} / \mathrm{sec})$, so that a veil would have advanced $\sim 0.5 \mu \mathrm{m}$ between succeeding frames. Such increments are readily detected (see Fig. 3) and are clearly distinguishable from a passive spread of the lamellar margin.

The filopodia examined were the "simple" filopodia, which differ morphologically (and likely functionally) from "mature" filopodia (Steketee and Tosney, 1999). Simple filopodia are of consistent diameter, rigid, and seldom harbor phase densities. They extend, support veils, and make adhesions. They can develop into mature processes that are larger in diameter (results of cytoplasmic engorgement or merging of two filopodia) and may branch (Steketee and Tosney, 1999).

For each filopodium that supports veils, the filopodial shaft was classified into three regions: one distal to the veil, one adjacent to and supporting the veil, and one comprising actin bundles that extend into the quiescent central region of the growth cone (Fig. 1). The region distal to the veil margin was easily distinguished. The region supporting the veil was defined as that region between the veil margin at fixation and the veil origin point at the filopodial base. The origin point was easily determined by assessing optical recordings and noting the site where the veil first 
emerged from the static, convex margin at the filopodial base. Moreover, the filopodial base itself coincided with a phase-dense spot that develops at each basal adhesion site before the filopodium emerges (Steketee et al., 2001). That spot remains static relative to the substrate and is confirmed by immunolocalization of marker in light microscopy or by the presence of a focal ring in stereo EM.

Shaft adhesions were detected by three criteria. In immunocytochemical analyses, shaft adhesions were defined as discrete puncta of either vinculin or Pty label (Fig. 2). In stereo EM, shaft adhesions were detected as "tethers" or spread areas attached to the substrate (see Fig. 4). In optical recordings, adhesions were inferred by comparing static and moving filopodia (Steketee et al., 2001). In recordings, filopodia that were moving laterally or vibrating were scored as nonadherent, whereas those that remained stationary for $\geq 1$ min were scored as adherent. In many cases, even portions of filopodial shafts that were moving or stationary could be detected. The shaft adhesions were clearly different from phase-dense "granules" in filopodia (which are rare in simple filopodia) (Steketee and Tosney, 1999), because the granules often move, but the adhesions do not.

To test whether veil advance correlated with shaft adhesions (Fig. 3), filopodia that were supporting veils at fixation were identified both in recordings and in corresponding fluorescent micrographs ( $n=197$ filopodia). To assure the most sensitive measure of the effect of adhesions, quantitative comparisons were made between regions of filopodial shafts that had or lacked veils. Each region was compared for filopodia on laminin alone and for filopodia on laminin whose tips had contacted guidance cues. Each region, with and without veils, was quantified separately by counting shaft adhesions as puncta of label per micrometer. Puncta were rounded, and although they varied in size, they were usually approximately the diameter of a filopodium. Puncta sometimes lay in close continuity or were connected by streaks of label (Fig. 2b), but even adjacent puncta usually presented as distinct bulges and could thus be easily counted. To facilitate direct comparisons, values were normalized. As the standard for comparison, we focused on filopodia that were not contacting guidance cues and used as the baseline the region of these filopodia that lacked veils. Thus, values were expressed as a percentage of the average adhesion frequency in regions lacking veils. Even in these regions, small gaps often lay between adhesions, so that a $100 \%$ value does not mean that every portion of the shaft was adherent. Indeed, the frequency of adhesions rose slightly above baseline among filopodia that contacted posterior sclerotome cells. Note that neither the basal adhesions nor the filopodial tip adhesions to cells were counted as shaft adhesions.

To determine whether veils commonly stopped advancing when they reached a shaft adhesion, the incidence of "terminal adhesions" (puncta of label within $1 \mu \mathrm{m}$ of the veil margin) was examined in veils that were actively advancing at fixation and in veils that had ceased to advance and stabilized without retracting within the last $30-60 \mathrm{sec}(n=208$ veils in 32 growth cones).

To assess roles of adhesions in process stability, filopodia were classed as having or lacking adhesions distal to the veil. Veils were considered stable if they remained at their maximum extent $\geq 1$ min before fixation and were considered to be retracting if they had receded. Lateral instability was displayed as one filopodium moved laterally and merged with another ( $n=62$ in 10 growth cones).

To distinguish between two alternatives, (1) that adhesion-free regions promote veil advance or (2) that veils remove adhesions as they advance, adhesions were experimentally decreased directly. During recording, an anti-laminin antibody (Sigma) was added at 1:100 or 1:500 dilution, and cultures were fixed and labeled as described above. As a control, equal volumes of carrier (medium) were added. Quantitative analysis focused on 26 veils from three growth cones treated at 1:100 dilution. To assess veil advance, the maximum distance that each veil advanced was measured before and after adding antibody. Postaddition means were normalized as a percentage of the preaddition mean. To assess veil initiation, the frequency of veils that had advanced $>2 \mu \mathrm{m}$ was measured per growth cone per minute, before and after the addition of antibody.

To assess the relationship between veil advance and the position of shaft adhesions, filopodia were classed by the patterns of shaft adhesions: along the entire shaft, confined to the proximal shaft, confined to the distal shaft, and absent. To determine whether veil advance varied consistently with pattern, the proportion of filopodia in each class that had advancing veils was determined for each growth cone, both on laminin and after the addition of antibody (1:100 dilution) ( $n=75$ filopodia in nine untreated growth cones; $n=78$ filopodia in six treated growth cones).

\section{RESULTS}

The relationship between veil advance and filopodial adhesions was analyzed in avian sensory neurons. Growth cones were optically recorded as they advanced on laminin and as they contacted two cell types, Schwann cells and posterior sclerotome, which guide growth cones in vivo (Son and Thompson, 1995; Tannahill et al., 1997) by regulating veil advance (Oakley and Tosney, 1993; Steketee and Tosney, 1999; Polinsky et al., 2000). Observations focused on simple filopodia (those that had yet to mature into thicker or branched processes).

\section{Dual criteria identify adhesions along filopodia}

Adhesions were identified in recorded growth cones using dual criteria: stability and marker localization. We developed a reproducible labeling assay to detect adhesions using a controlled extraction that retains vinculin and Pty only when they have been stabilized by adhesion (see also Steketee et al., 2001). Vinculin localizes to adhesion sites in other cells (for review, see Schoenwaelder and Burridge, 1999), and Pty signaling at adhesions is important to both cellular and growth cone motility and guidance (Wu and Goldberg, 1993; Goldberg and Wu, 1996; Desai et al., 1997; Dogic et al., 1999; Renaudin et al., 1999).

Adherent filopodial regions could be reliably inferred from their stability relative to the substrate in optical recordings, and the inferred adhesions accorded well with adhesions detected by vinculin and Pty localizations. Along simple filopodia, adherent regions were stable with respect to the substrate, whereas nonadherent regions exhibited obvious vibratory and/or lateral movements (Fig. 2). The entire filopodial shaft might be stationary (Fig. 2a,b), or the entire shaft might move (Fig. $2 c, d$, arrows). Even local adhesions were detectable: some filopodia exhibited both adherent and nonadherent regions (Fig. $2 e-g$ ). For instance, the proximal shaft could move, whereas the distal shaft remained stationary and resisted lateral tension exerted by the moving proximal shaft (Fig. 2e,g). The correlation between stationary regions and filopodial adhesions could be remarkably precise. For instance, a shaft could even pivot about a stationary point that labeled on fixation for an adhesion marker (Fig. 2f). Because stationary regions accord well with both adhesion markers, both label and motile histories are useful criteria to identify adherent and nonadherent sites.

Regardless of whether the shaft was adherent, the bases of virtually all filopodia both remained stationary and labeled for adhesion markers (Fig. $2 b-d$ ). These basal adhesions characterize $\sim 98 \%$ of all filopodia, regardless of filopodial or veil activities, and play specific roles in filopodial initiation (Steketee et al., 2001). Unlike shaft adhesions, whose maximum lifetime is necessarily that of a filopodium $(6.2 \pm 0.8 \mathrm{~min})$ (Steketee and Tosney, 1999), basal adhesions appear to be long lived, emerging before filopodia emerge and remaining in place as the growth cone advances, until they are occluded by the dense central region. Basal adhesions are excluded from analysis in the current study.

Based on motile analysis, shaft adhesions appear to develop de novo along the shafts and do not represent previous tip contacts that are retained as a filopodium elongates. During the period when filopodia are emerging, they are very seldom adherent, even at their tips; they consistently adhere only at their bases (Steketee et al., 2001). Once elongated, many filopodia that are observed to 

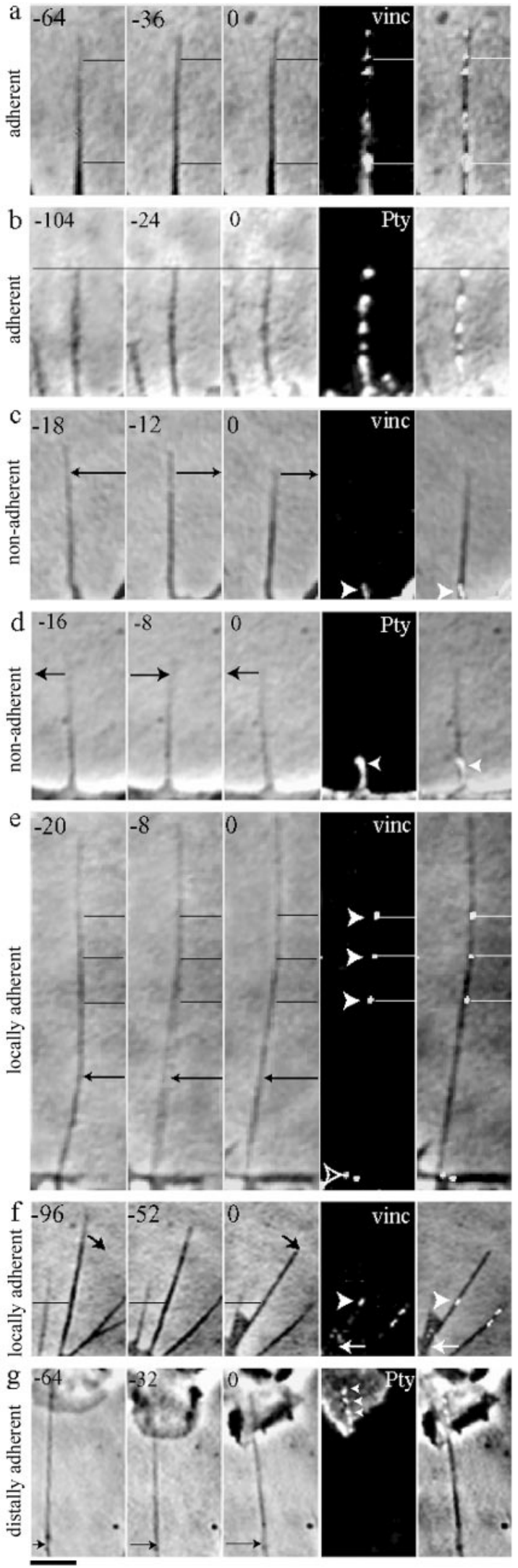

Figure 2. Stationary sites on filopodia accord with punctate adhesions detected by the adhesion markers vinculin (vinc) and Pty. $a, b$, Stationary filopodia exhibited adhesions along their length. $c, d$, Moving filopodia exhibited adhesions restricted to basal adhesions at stationary filopodial bases. $e, f$, Stationary sites along moving filopodia accorded with punctate move laterally (and thus lack stable adhesions at either their tip or along their shaft) do make obvious shaft and tip adhesions as they contact the substrate. The most convincing evidence comes from filopodia that contact posterior sclerotome cells. Most such filopodia assessed were obviously moving before their tips contacted the cell and then contacted the cell only at their tips. Nonetheless, abundant shaft adhesions consistently developed (see below). It is thus unlikely that shaft adhesions represent former tip adhesions.

\section{Veils advance preferentially along filopodial regions that lack adhesions}

On laminin, veils advanced selectively down regions of filopodia that were free of shaft adhesions (Fig. 3). Excluding the filopodial base, puncta of label indicating adhesions were sparse or absent along the proximal filopodial region directly opposed to an advancing veil (Fig. 3a-c, open arrowheads). Veil regions were also clearly nonadherent by motile criteria, because the proximal filopodial shafts often moved during veil advance (Fig. $3 b$, arrows). Adhesion-free regions along filopodia were closely linked to veil advance both temporally and spatially. In contrast, filopodia without veils were commonly stationary and had abundant adhesions. These qualitative observations are supported by quantitative analysis of adhesion frequencies in regions of shafts with and without veils. Regions with veils consistently had fewer puncta of label per micron than did regions without veils (Fig. $3 d, e)(p \leq 0.001)$. The close correlation between adhesion-free regions and veil advance suggests that shaft adhesions inhibit veil advance.

Veils that had stopped advancing and stabilized also showed features consistent with the premise that shaft adhesions inhibit veil advance. Veils in two categories were analyzed: those that were actively advancing just before fixation and those that had recently ceased to advance. Each class was scored for terminal adhesions, puncta of label on filopodia within $1 \mu \mathrm{m}$ of the veil margin at fixation. Terminal adhesions were common (40 of 55; $73 \%$ ) at the margin of veils that had just ceased advance but were rare $(6$ of $49 ; 12 \%)$ at margins of veils that had been advancing actively ( $p<0.01 ; n=104$ veils in 16 growth cones). These results suggest that veils cease their advance as they encounter shaft adhesions.

\section{Third criterion for detecting shaft adhesions supports an inhibitory relationship with veil advance}

Although detergent stability of adhesion markers is an accepted criterion for adhesions (Meiri and Burdick, 1991), an additional criterion for shaft adhesions was sought that did not require selective extraction and that preserved structure well even in unattached filopodia. Although various adhesions can be visualized with interference reflection microscopy, this method was not chosen for two reasons. First, it can fail to distinguish between transient, vinculin-negative point adhesions and more stable vinculin-positive point adhesions in growth cones (Arregui et al., 1994; Renaudin et al., 1999). Second, it selectively reveals portions of the cell that are close to the substratum, so that elements

filopodial adhesions (guidelines), whereas label was undetectable along moving regions (arrows). Note that a filopodium can pivot about a punctate adhesion site ( $f$, arrowhead). $g$, A filopodial shaft moved laterally (arrow) and lacked label, whereas the tip contacting the Schwann cell remained stationary and displayed adhesion label. Seconds before fixation are indicated at the top left of each panel. The far right column overlays label and the last phase frame. Scale bar, $1 \mu \mathrm{m}$. 

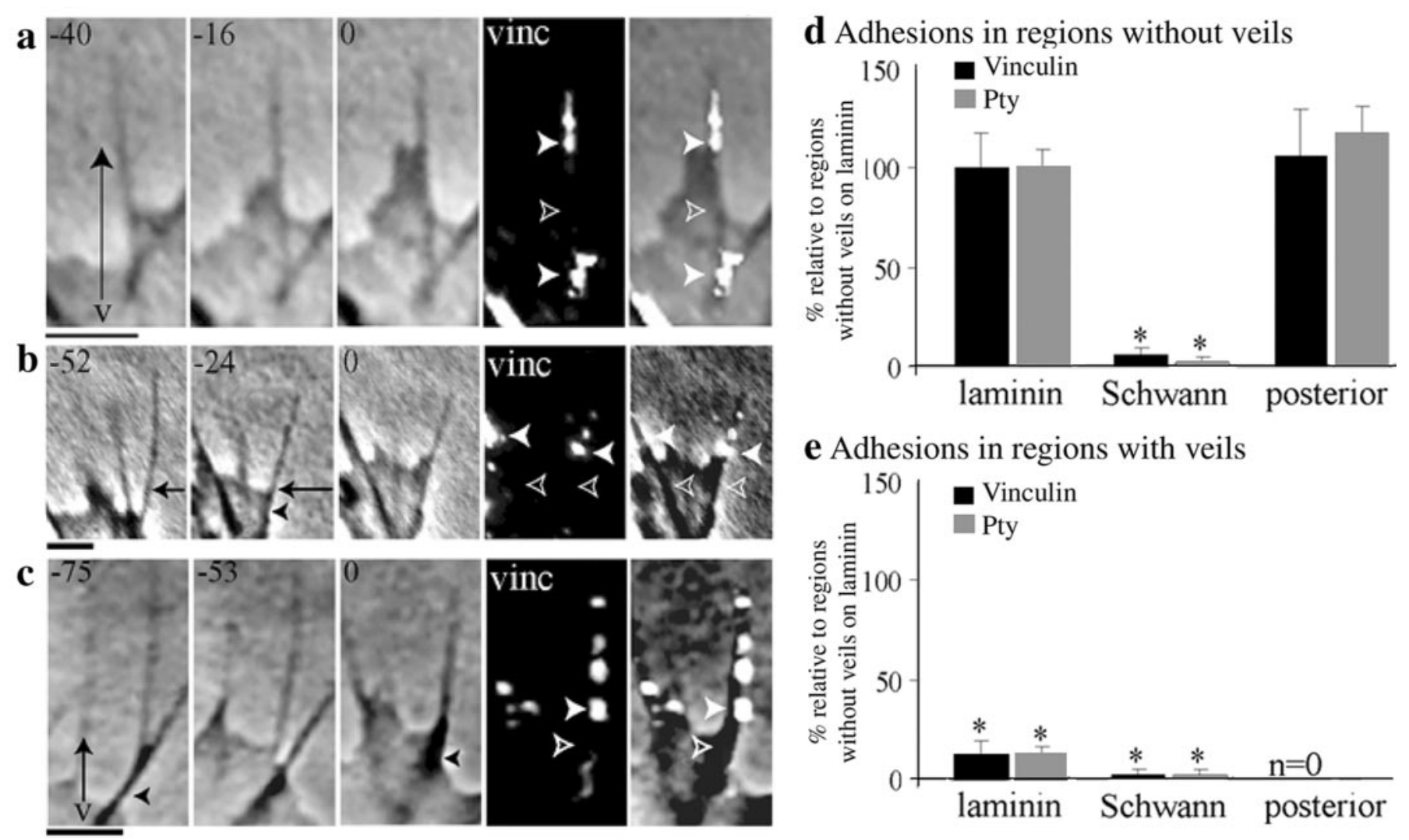

\section{e Adhesions in regions with veils}

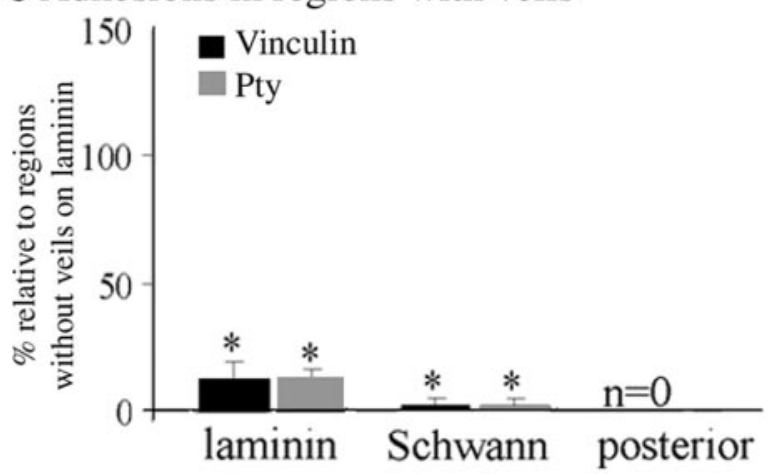

Figure 3. Veils advance down filopodial regions that lack adhesions. $a-c$, When veils advanced, filopodial adhesions ( filled arrowheads) were confined to bases and distal shafts, and the veils $(v)$ advanced along shaft regions that lacked detectable shaft adhesions (open arrowheads). These regions were often clearly nonadherent by motile criteria as well, because they changed position from frame to frame $(b$, arrows $)$. Seconds before fixation are indicated at the top left of each panel. vinc, Vinculin. Scale bars, $1 \mu \mathrm{m} . d, e$, To assess shaft adhesions, filopodia supporting veils were assessed. Two regions of their filopodial shafts were quantified separately, as puncta of label per micrometer: $d$, regions without veils; $e$, regions with veils. As a baseline for both graphs, the region without veils was used from filopodia whose tips contacted laminin (rather than cells); all values were normalized to the average from this region (mean \pm SEM: vinculin, $0.53 \pm 0.06 \mathrm{puncta} / \mu \mathrm{m} ; \mathrm{Pty}, 0.69 \pm 0.05 \mathrm{puncta} / \mu \mathrm{m})$. When filopodia are on laminin and their tips are not contacting cells, then the regions without veils had abundant shaft adhesions $(d)$, whereas regions with veils had few shaft adhesions $(e)$. When filopodia contacted Schwann cells, both the regions with veils and regions without veils virtually lacked shaft adhesions. Conversely, when filopodia contacted posterior sclerotome cells, no veils were detected, and shaft adhesions were abundant. ${ }^{*} p \leq 0.001$ compared with baseline regions without veils. $n=100$ filopodia (Vinculin), 100 filopodia (Pty). Note that basal adhesions were excluded, as were tip adhesions made to cells.

distant from the substratum are visualized poorly if at all, including many newly extending filopodia and their veils.

An independent criterion was supplied by whole-mount stereo EM. This approach preserves the structure of even nonadherent filopodia well. It has been used previously to reveal tethers of filamentous material that extended from growth cone surfaces to the substrate in association with adhesive loci (Tsui et al., 1988).

To assess the utility of stereo EM as a criterion for shaft adhesions, filopodia that were moving or static in the last minute before fixation were compared (Fig. 4a-d). All static filopodia $(n=12)$ displayed obvious associations with the substrate. These adhesive specializations were either filamentous tethers (Fig. 4a) or broad spread areas of membrane that were clearly attached to the substrate (Fig. 4b). All moving filopodia $(n=20)$ lacked these specializations, even when they had fallen and come to lie on the substrate during fixation. Some were suspended above the substrate (Fig. 4c), whereas those that lay on the substrate (possibly having fallen there during processing) all had even margins without detectable spread areas or tethers (Fig. $4 d$ ).

Stereo EM analysis also detected a consistent relationship between shaft adhesions and veil advance. Shaft adhesions indicated by tethers or spreading were detected just distal to veils that had recently stopped (Fig. $4 e)(n=14$ veils) but not distal to advancing veils (Fig. $4 f)(n=25$ veils). The lack of tethers distal to advancing veils was not caused by deviations in fixation that prevented their detection, because tethers were often detected along other filopodia on the same growth cone, as well as at the tips of filopodia that were supporting veil advance (Fig. $4 f$ ).

\section{Filopodia and veils show lateral instability in the absence of adhesions}

When a pair of filopodia support a veil, the veil and filopodia commonly show one of three behaviors (Fig. $5 a-c$ ). (1) They may merge to form a single, thicker process. After merging, this process usually elongates (Fig. $5 a$ ) as though it had received materials necessary for further protrusion. Generally, merging is a consequence of lateral instability, in which one filopodium moves laterally, as though a force exerted by the veil pulled the processes together. Multiple merging events lead to a thickened process with prominent adhesions (Fig. $5 d$ ), consistent with early stages in neurite formation. (2) The veil and filopodia may stabilize. Veils that have stabilized assume a convex profile, remain extended, and may ultimately engorge with cytoplasm. (3) Veils may retract, retreating proximally toward the growth cone body. 

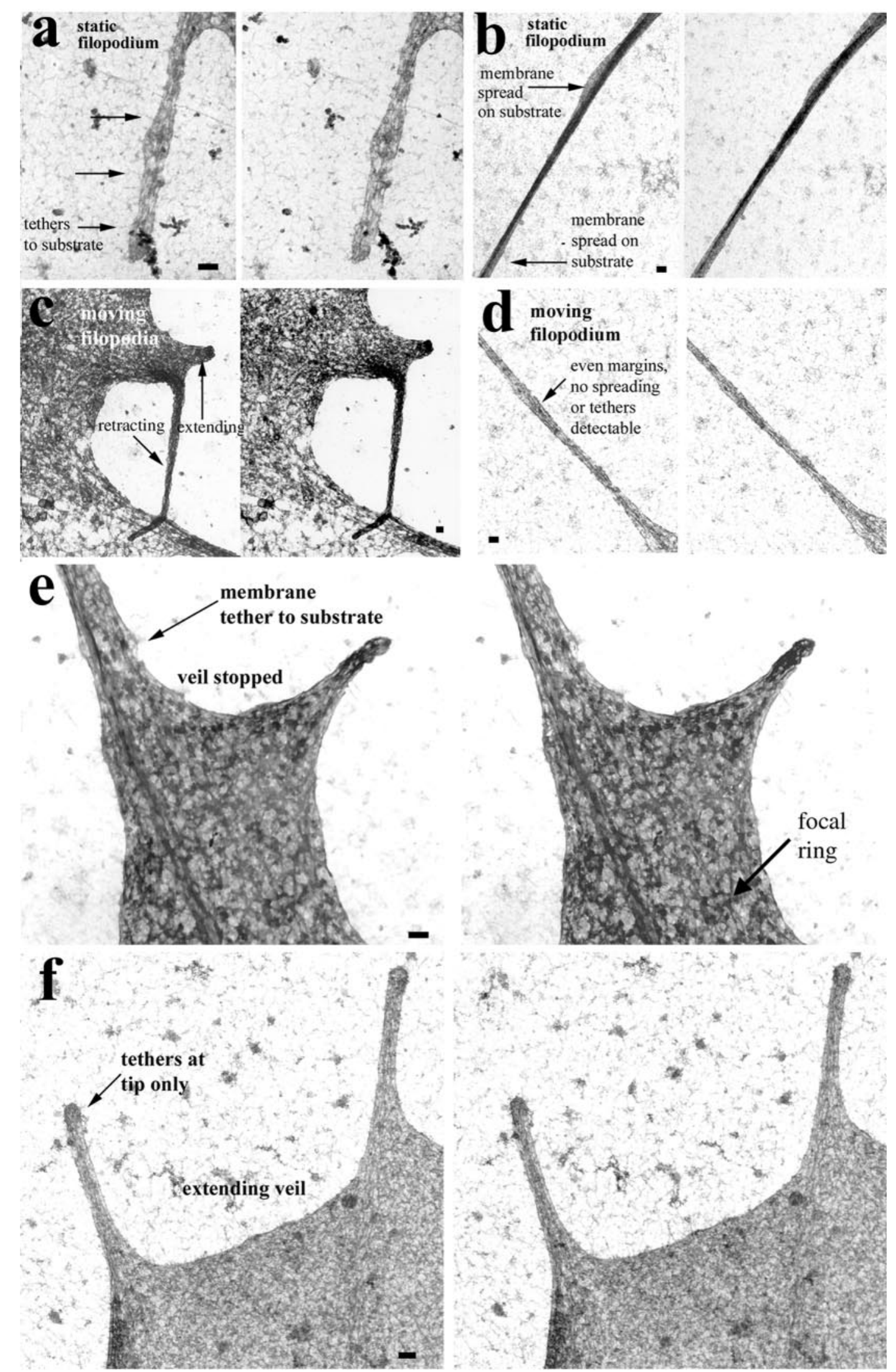

Figure 4. Stereo EM criteria identify shaft adhesions and support an inhibitory relationship between shaft adhesions and veil advance. Filopodia that were static $(a, b)$ or moving $(c, d)$ in the last minute before fixation were compared using whole-mount stereo EM. Static filopodia clearly displayed attachments to the substrate. $a$, A static filopodium that displays multiple filamentous tethers to the substrate (arrows). $b$, A static filopodium that displays a membranous spread area that is clearly attached to the substrate. In contrast, moving filopodia lacked obvious association (Figure legend continues.) 

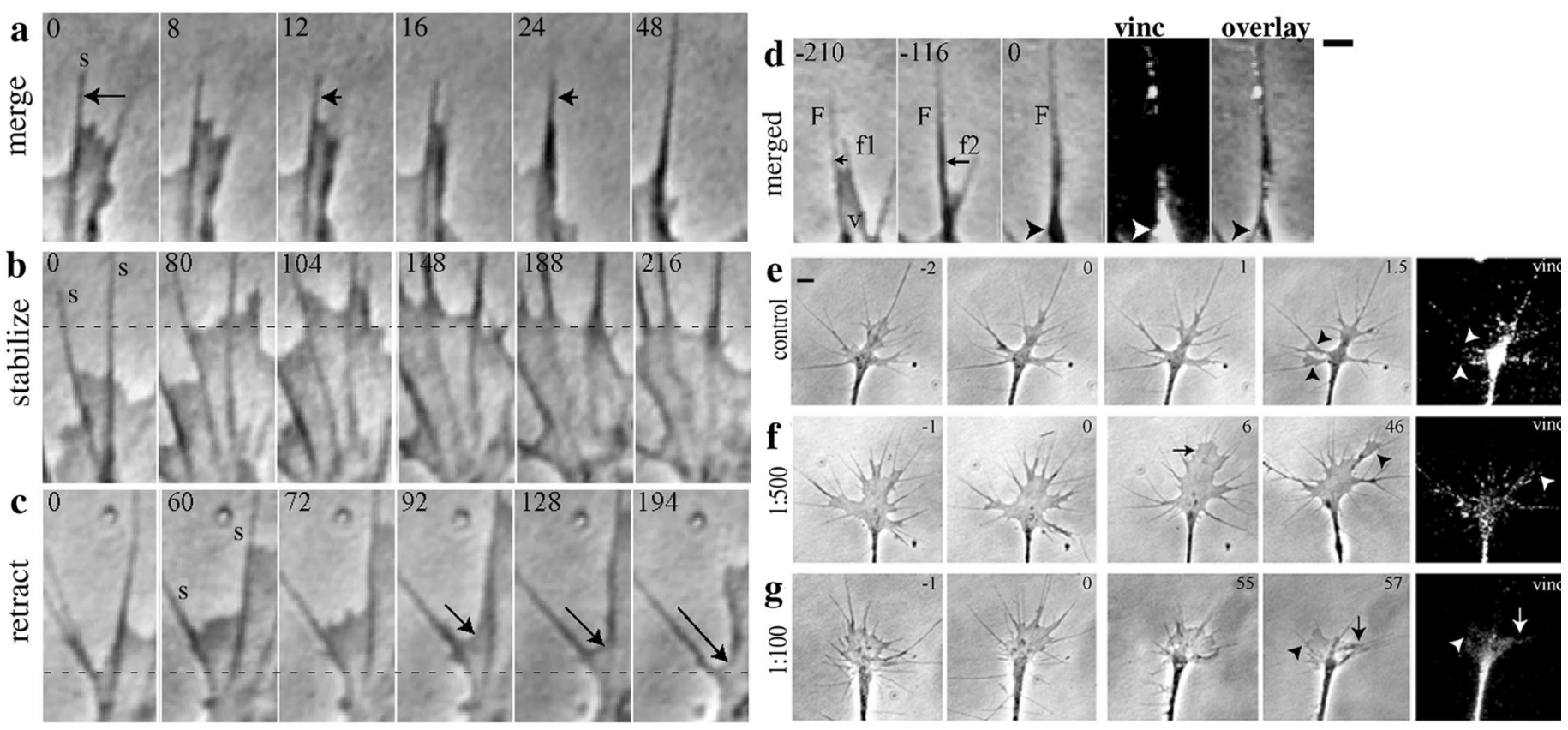

Figure 5. Veil and filopodial stability on laminin $(a-d)$ and after reduction of adhesion with anti-laminin antibody $(e-g) \cdot a-d$, Stably adherent filopodia $(s)$ were easily distinguishable by motile criteria from moving filopodia (arrows, $a, d$ ). $a$, When only one filopodium was adherent, the veil and nonadherent filopodium moved toward the adherent filopodium, and the processes merged. Typically, the thickened filopodium then elongated. When both filopodia were adherent, the veil either stabilized $(b)$ or retracted $(c$, arrow). Dotted lines in $b$ and $c$ indicate the same position relative to the substrate in each frame. $d$, Merged regions commonly displayed large puncta of adhesion label. Time in seconds is indicated at the top left of each panel. $v$, Veil; $F$, adherent filopodium; $f 1$, first filopodium to merge; $f 2$, second filopodium to merge. Scale bar, $1 \mu$ m. $e-g$, As adhesion was reduced with anti-laminin antibody, growth cones rapidly became more lamellar, and process stability decreased. In accord with a control by shaft adhesions, veils still advanced preferentially along nonadherent regions of filopodia (arrowheads). $f, g$, Antibody addition at time 0 obviously decreased the intensity and distribution of label. $f$, At 1:500, the leading edge of the growth cone showed a transient increase in lamellar expanse. $g$, At 1:100, large lamellar expanses advanced even after long incubation $(t=55 \mathrm{~min}$ ), but processes were unstable and tended to merge (arrows). Data shown were collected and presented under the same conditions at the same time. Time in minutes is indicated at the top right of each panel, relative to the addition of carrier $(e)$ or antibody $(f, g)$ at time 0 . Scale bar, $10 \mu \mathrm{m}$. The far right columns overlay label and the last phase frame. vinc, Vinculin.

Although retraction did not obviously correlate with adhesions, lateral stability and merging were very sensitive to adhesions. Any adhesion, at the tip, shaft, or base, appeared to suffice to prevent lateral movement of that portion of the filopodium, simply by tethering the filopodium to the substrate (Table 1). Loss of lateral stability was most common among filopodial pairs supporting veils, consistent with the advancing veil exerting a force to bring filopodia together. If one supporting filopodium lacked adhesions, then that filopodium and the veil moved laterally and merged with the adjacent adherent filopodium. When both basal and proximal adhesions were absent, filopodia could merge proximally. When both supporting filopodia were adherent, the veil either stabilized or retracted. These results suggest that filopodial adhesions at all three sites restrict lateral mobility.

\section{Experimentally reducing adhesion alters veil advance by changing shaft adhesion patterns}

The consistent relationship between shaft adhesions and veil advance could arise by two different mechanisms. Veils might remove shaft adhesions as they advance. Alternatively, veils might advance preferentially where shaft adhesions are absent. To dis-

\begin{tabular}{|c|c|c|c|}
\hline Distal adhesions & $\begin{array}{l}\text { Processes } \\
\text { merged }\end{array}$ & $\begin{array}{l}\text { Veils } \\
\text { stabilized }\end{array}$ & $\begin{array}{l}\text { Veils } \\
\text { retracted }\end{array}$ \\
\hline On both filopodia & $2 / 41$ & $14 / 14$ & $7 / 7$ \\
\hline On one filopodium & $39 / 41$ & $0 / 14$ & $0 / 7$ \\
\hline
\end{tabular}

Filopodial pairs supporting a veil were classed as having or lacking shaft adhesions distal to the veil (shaft adhesions were always absent on that portion of the shaft that was adjacent to the veil), and the fate of processes was assessed using the following criteria. Retracted veils had receded from their maximum projection before fixation. Stabilized veils had stopped advancing and remained at their maximum projection for $\geq 1$ min just before fixation. Merged processes resulted from lateral movement and a combination of processes. Distal adhesions did not predict whether veils stabilized or retracted. However, when one filopodium lacked distal adhesions, it consistently moved laterally and merged. In two cases, a filopodium had distal adhesions but lacked both proximal shaft adhesions and basal adhesions. In both cases, the proximal shaft and base moved laterally and merged (top left column), but the distal portion of the filopodia did not, suggesting that lateral mobility is restricted by adhesions at proximal as well as at distal sites. ( $n=62$ filopodial pairs in 10 growth cones).

tinguish between these alternatives, and to test how shaft adhesions are related to veil initiation, veil advance, veil stopping, and lateral stability, growth cones were treated during optical record-

$\leftarrow$

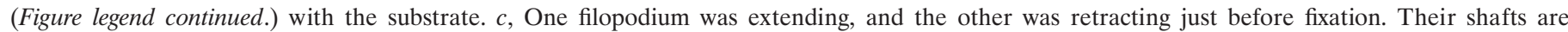

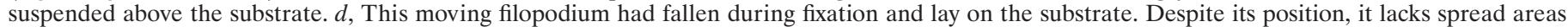

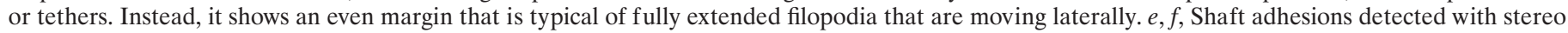

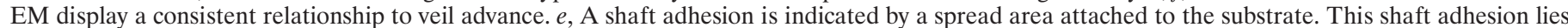

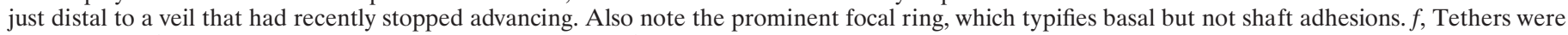
detected at a filopodial tip but not along the shaft of these filopodia that were supporting active veil extension. Scale bars, 100 nm. 

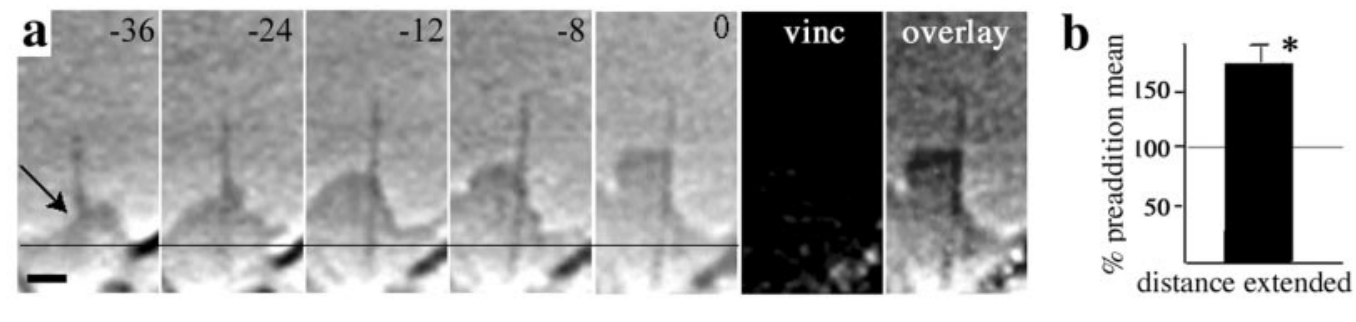

\section{c Frequency of veil advance}
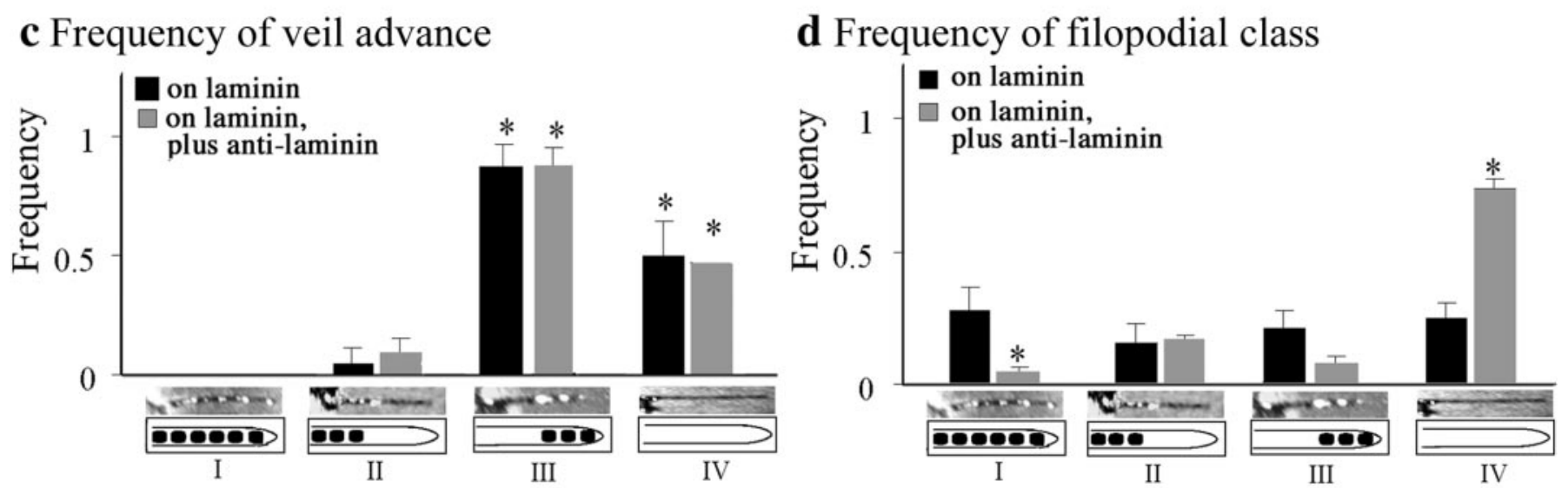

Figure 6. Veil advance accords with shaft adhesion patterns even when overall adhesion is reduced with antibody. $a$, $b$, Decreasing filopodial adhesion increases the distance that veils travel down filopodia. $a$, Typical example: in the presence of 1:100 anti-laminin antibody, this filopodium lacked adhesions and supported robust veil advance. Time before fixation in seconds is indicated at the top right of each panel. vinc, Vinculin. Scale bar, $1 \mu \mathrm{m}$. $b$, Compared with the preaddition mean, the distance veils advancing down filopodia increased after treatment with 1:100 anti-laminin. Mean \pm SEM: $173 \pm 17 .{ }^{*} p<0.05 . n=26$ veils from three growth cones. $c, d$, Filopodia were classified by the pattern of adhesions along their shafts (excluding the consistent basal adhesion). Class: $I$, Adhesions along the entire shaft; $I I$, adhesions confined to the proximal shaft; $I I I$, adhesions confined to the distal shaft; $I V$, no adhesions. Each pattern is indicated by a micrograph (Pty label) and a corresponding schematic. $c$, Frequency of veil advance. Within each adhesion class, the proportion of filopodia with advancing veils was determined for each growth cone. In filopodia lacking proximal adhesions (classes III, IV), veils advanced more readily than in those having proximal adhesions (classes I or II; ${ }^{*} p \leq 0.01$ ), either with or without anti-laminin antibody. $d$, Frequency of filopodial class. Decreasing adhesion with 1:100 anti-laminin antibody shifted the population distribution toward the class that lacked adhesions. The proportion of filopodia within each class was determined for each growth cone. Untreated growth cones (black bars) had equivalent proportions of filopodia in each class, showing that their differential veil advance in $c$ is not attributable to different numbers of filopodia in each adhesion class. Compared with the untreated condition, growth cones treated with anti-laminin (gray bars) had significantly fewer filopodia that were fully adherent (class I; $p<0.05$ ) and significantly more filopodia that lacked adhesions (class IV; $p \leq 0.0001$ ). Graphs show means \pm SEM per growth cone ( $n=75$ filopodia in 9 untreated growth cones; $n=78$ filopodia in 6 treated growth cones).

ing with anti-laminin antibodies to reduce substrate adhesions directly. If shaft adhesions do control veil advance and lateral stability, then reducing adhesion should facilitate veil advance and increase lateral instability, without altering veil initiation.

Qualitatively, anti-laminin antibodies coordinately reduced filopodial adhesions (as monitored by both optical and immunocytochemical criteria) and altered growth cone morphology, producing growth cones with a more lamellar form (Fig. 5e-g). When antibody was added, growth cones rapidly developed prominent veils, producing a more lamellar form, consistent with a stimulation of veil advance. Moreover, when adhesion was reduced, veils and their filopodia showed extensive lateral instability, and merging events were common. However, the relationship between veil advance and shaft adhesions on individual filopodia was retained. Despite the overall reduction in adhesion, veils still advanced preferentially down regions of filopodia that lacked shaft adhesions (Fig. 5f,g, arrowheads).

The correlation between shaft adhesions and lack of veils might be explained by an alternative mechanism, in which shaft adhesions inhibit veil initiation instead of inhibiting veil advance. If shaft adhesions do selectively control advance rather than initiation, then altering adhesion should leave veil initiation unchanged. Before antibody treatment, the frequency of veils initi- ating per growth cone per minute was $0.28 \pm 0.06$. Anti-laminin antibody increased the initiation frequency, but not significantly $(0.41 \pm 0.05 ; p=0.1)$. The apparent increase likely results from incipient veils advancing farther, rather than initiating more often. To be counted as initiating, a veil had to have advanced $>2$ $\mu \mathrm{m}$, and without antibody, some incipient veils typically fail to meet this minimal criterion. More veils would be scored if they advanced farther after treatment.

If shaft adhesions stop veil advance when the veils encounter them, then the distance that veils advance should depend on the prevalence of shaft adhesions. Reducing adhesions should allow veils to advance farther. Experimentally decreasing adhesion did increase how far veils advanced (Fig. $6 a, b$ ). The maximum distance that individual veils advanced was measured before and after addition of anti-laminin antibody. Compared with the preaddition mean, veils advanced farther down filopodia when adhesion was reduced (mean $\pm \mathrm{SEM}: 173 \% \pm 17 ; p<0.05 ; n=26$ veils from three growth cones).

If veil advance is controlled by shaft adhesions, then the proximal-distal pattern of adhesions should be vital, because veils progress along the filopodial shafts from proximal to distal. Filopodia with proximal adhesions should lack veils, regardless of 
whether they have distal adhesions. Conversely, filopodia that lack proximal adhesions should regularly support veils, again regardless of whether they have distal adhesions. Altering the degree of adhesion should not alter this dependent relationship.

Advance is indeed a function of proximal-distal adhesion patterns, regardless of the overall degree of substrate adhesion (Fig. 6c). In untreated growth cones, veils advance down filopodia that lack proximal shaft adhesions (classes III and IV) significantly more often then they advance down filopodia that have proximal shaft adhesions (classes I and II; $p<0.01$ ). The importance of proximal adhesions was unaltered by the presence of distal adhesions, ruling out long-range effects (compare class I with class II and class III with class IV). Moreover, although treatment with anti-laminin antibody reduced adhesion generally (Fig. $5 f, g$ ), it did not alter the dependent relationship between veil advance and shaft adhesion pattern (Fig. $6 c$ ). Even in the presence of antibody, veils advanced predominantly down filopodia lacking proximal adhesions $(p<0.01)$. Veil advance is thus related to the pattern, rather than to the overall degree, of adhesion.

Decreasing adhesion with antibody alters veil advance in growth cone populations by shifting the population distribution toward filopodia that lack shaft adhesions (Fig. $5 d$ ). Compared with untreated growth cones, those treated with anti-laminin had significantly fewer filopodia that were fully adherent $(p<0.05)$ and significantly more filopodia that lacked shaft adhesions $(p<$ $0.0001)$. These observations show that veil advance is not a product of overall adhesion and is not related to an overall inhibition of integrin signaling by the antibody. The effects are not global. Instead, the advance of a veil is related directly to the pattern of shaft adhesions along its supporting filopodium.

\section{Cellular cues detected by tip adhesions alter shaft adhesions and veil advance coordinately}

When filopodia are contacting only one substratum, laminin, distinctive properties of tip versus shaft adhesions cannot be easily discriminated. However, such properties are revealed when the tip and shaft of an individual filopodium contact different substrates. Analyzing contact with either of two natural substrates, Schwann cells or posterior sclerotome cells, had disclosed previously that a signal received by a tip adhesion systematically alters veil advance down the shaft of the contacting filopodium. Schwann cells stimulate veil advance (Polinsky et al., 2000), whereas posterior sclerotome cells prohibit veil advance (Oakley and Tosney, 1993; Steketee and Tosney, 1999). The responses are stereotyped, rapid, and robust, virtually $100 \%$, so that the fate of veils on every filopodium that adheres to a cell is predictable. Each response is also highly discrete, altering aspects of veil dynamics selectively. For instance, contact with posterior sclerotome alters veil advance without altering veil initiation. Veils initiate at the same rate but fail to advance down contacting filopodia, as though the filopodia fail to support veil advance. These physiologically relevant interactions thus make ideal assays for identifying elements that control veil advance. Therefore, we asked whether filopodia contacting these cells altered veil advance and shaft adhesions in concert.

The inhibitory relationship between shaft adhesions and veil advance was dramatically reproduced during interactions with these cells. Filopodia contacting cells that inhibit veil advance were found to form abundant shaft adhesions. When the filopodial tips contacted posterior sclerotome, the filopodial shafts became adherent to the laminin on three criteria: they were
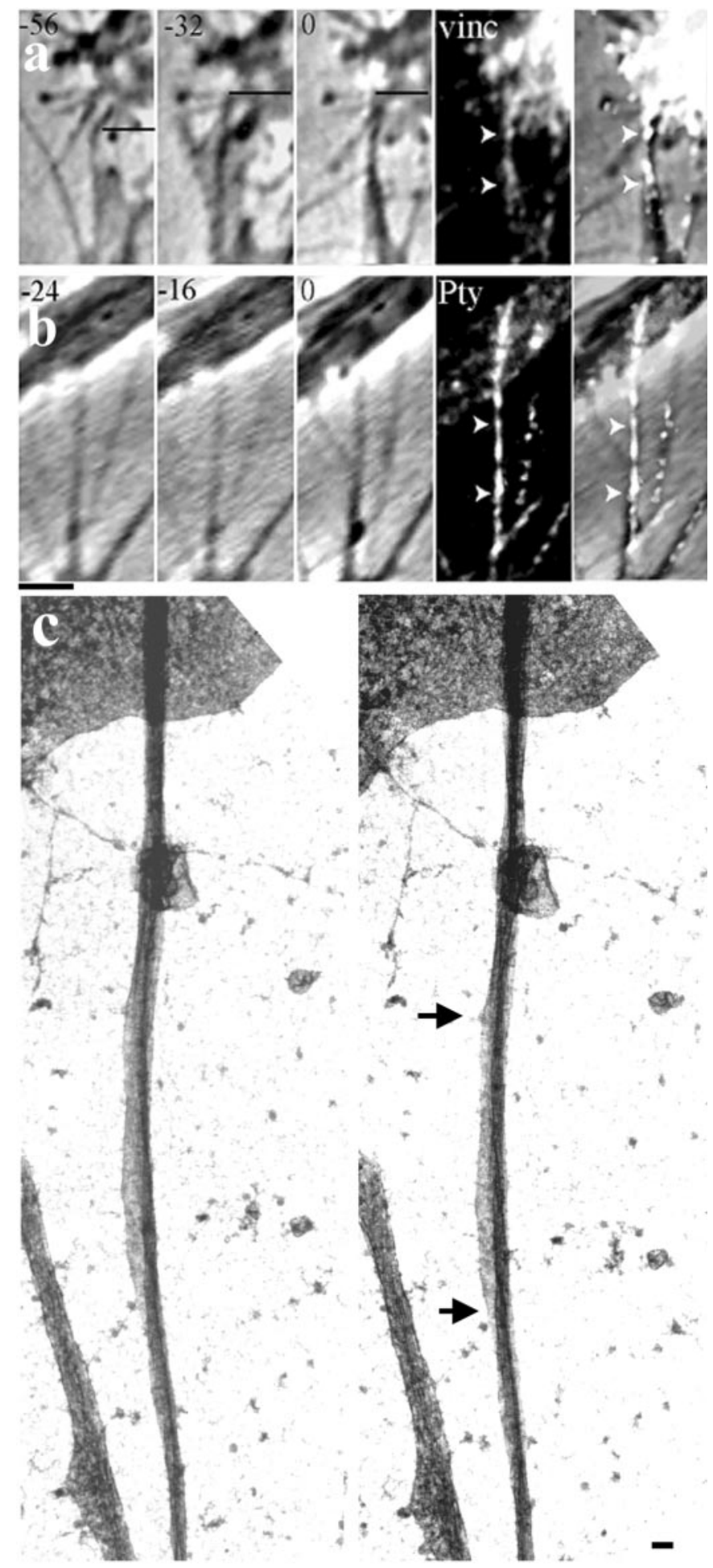

Figure 7. Guidance cues that inhibit veil advance coordinately stimulate shaft adhesions. $a-c$, When filopodial tips contacted posterior sclerotome cells, veils failed to advance, and the contacting filopodia displayed multiple shaft adhesions. $a, b$, Even filopodia that first stably contacted the cell seconds before fixation exhibited robust label (arrowheads). Time in seconds is indicated at the top left of each panel. The reference line in $a$ indicates the filopodial tip, which became stationary (stably adherent) 32 sec before fixation. vinc, Vinculin. $b$, Two filopodia contacted the cell; the one on the right contacted in the last frame before fixation. $c$, In stereo $\mathrm{EM}$, a filopodium contacting a posterior sclerotome cell displays a broad region of membrane attachment to the substrate (between arrows). Scale bars: $a, b, 1 \mu \mathrm{m} ; c, 0.1 \mu \mathrm{m}$. 
stationary, exhibited robust vinculin and Pty labels, and displayed adhesive specializations to the laminin substrate in stereo EM (Fig. $7 a-c$ ). Adhesion was detected immediately after contact and thus was rapid enough to account for the rapid inhibition of veil advance on contact. Because posterior sclerotome cells inhibit veil advance so robustly, there were no regions that had veils. Likewise, all of the filopodia contacting these cells had abundant shaft adhesions. The frequency of shaft adhesions did not differ from that found on the adherent regions of filopodia on laminin alone, which also failed to support veils (Fig. 3d,e). Thus, the signal stimulated by contact with this cellular cue appears to inhibit veil advance by promoting shaft adhesions to laminin.

In contrast, filopodia-contacting cells that induce veil advance displayed minimal or no shaft adhesions. When a filopodial tip contacted a Schwann cell, the filopodial shaft was nonadherent to the laminin substrate on three criteria: the shafts were often moving (Fig. $2 g$ ), the shafts lacked vinculin or Pty label (Fig. $8 a-d)$, and when viewed in stereo electron micrographs, the filopodial tips were closely apposed to the cell, but the shafts arched above the substrate and lacked adhesive specializations visible in stereo EM (Fig. 8e).

The absence of shaft adhesions was spatially independent of veil advance. The entire shaft overlying the laminin, rather than just the region with veils, generally lacked adhesions. Quantitatively, compared with filopodial regions on laminin that lacked veils, shaft adhesions were significantly fewer in regions with veils, and remarkably, shaft adhesions were also significantly fewer even in regions that lacked veils (Fig. $3 d, e)(p \leq 0.001)$. Adhesions were restricted to the filopodial base and to shaft regions directly opposed to the Schwann cell, suggesting that the ability of the filopodia to develop other adhesions was intact, that basal adhesions did not inhibit veil advance, and that adhesion of the tip to the cell altered adhesions to laminin specifically.

The time course was consistent with a rapid change in shaft adhesion that in turn enabled veil advance. Shaft adhesions were excluded early after Schwann cell contact, even before veils initiated and advanced (Fig. $8 a$ ). The lack of shaft adhesions persisted and coincided with the full period of veil advance (Fig. 8b). Veil advance ceased as filopodia begin to visibly thicken and develop adhesions, as expected because thickened filopodia on laminin rarely support veil advance and commonly exhibit robust adhesions (Fig. 5d). However, even when filopodia contacting Schwann cells merged, adhesions were still minimal (Fig. 8c). Adhesions became extensive only after the contacting filopodium began to form a neurite-like process (Fig. $8 d$ ). Because shaft adhesions were absent before veil advance and were very sparse even in regions without veils, shaft adhesions are not removed by veils during Schwann cell contact. Instead, the absence of adhesion prefigures and supports veil advance. These observations support the idea that filopodial contact with a Schwann cell initiates a signal that specifically prevents that filopodium from developing shaft adhesions on laminin and that the lack of shaft adhesions explains the robust veil advance.

Figure 8. Guidance cues that stimulate veil (v) advance coordinately inhibit shaft adhesions. On filopodia contacting Schwann cells, shaft adhesions were undetectable along the portion of the shaft that arches over the laminin substrate (open arrowheads). Shaft adhesions were undetectable when contacting filopodia before veils advanced $(a)$, as well as during veil advance $(b)$, even when other filopodia in the same field displayed robust adhesions ( filled arrowheads in $a$ ). Shaft adhesions (filled arrowheads) were seen when contacting filopodia only as they matured to form nascent neurites by merging with adjacent veils $(c)$ and filopodia (arrow)

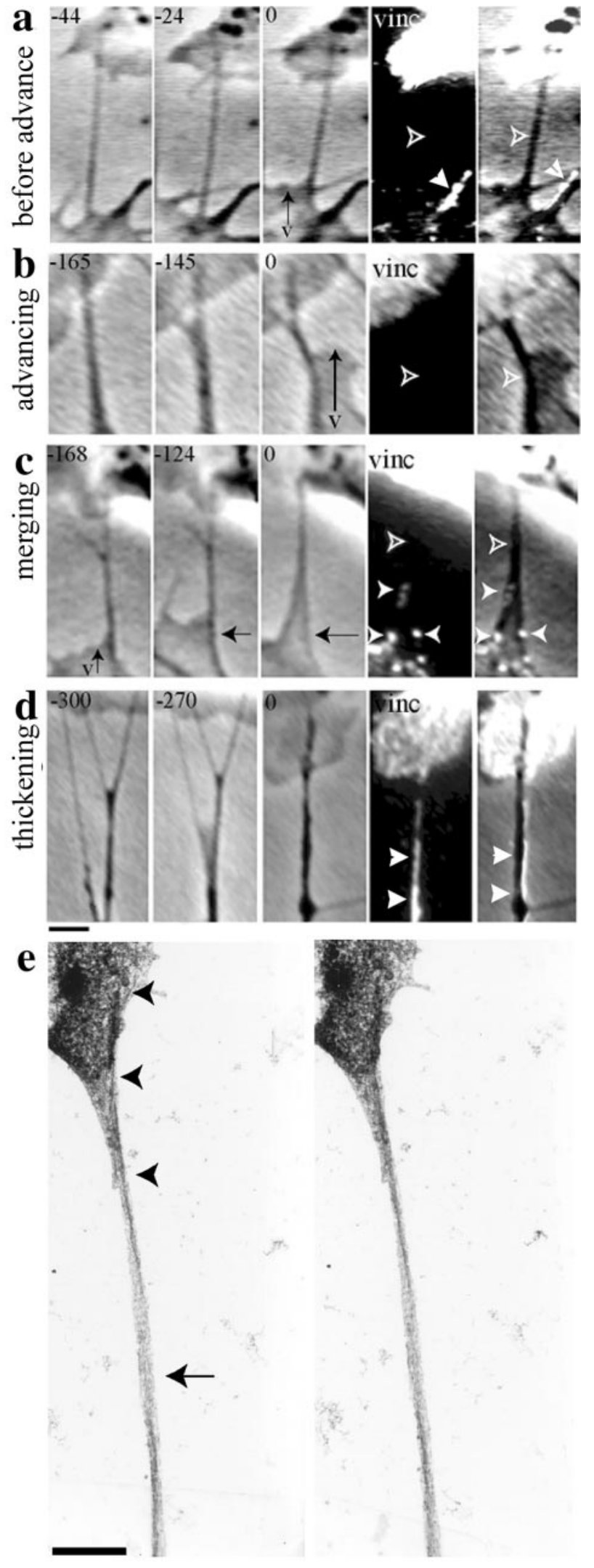

and thickening after multiple merges and/or engorgement with cytoplasm (d). vinc, Vinculin. $e$, Stereo EM shows a filopodial tip that had closely contacted a Schwann cell (arrowheads), whereas the shaft lay above the laminin substrate (arrow). In the phase frames, the seconds before fixation are indicated at the top left of each panel; far right columns overlay the label and the last phase frame. Scale bars, $1 \mu \mathrm{m}$. 


\section{DISCUSSION}

\section{Shaft adhesions control veil advance}

This study provides compelling evidence that shaft adhesions control veil advance. As monitored by optical recordings, adhesion markers, and electron microscopy, veils fail to advance down regions of filopodial shafts that adhere to the laminin substrate and instead advance preferentially down regions that lack shaft adhesions. In contrast, filopodial adhesions at the tip, shaft, and base were vital for lateral stability, to prevent veils and filopodia from moving laterally and merging with adjacent, adherent filopodia. These correlations were validated by experiments that reduced substrate adhesion directly. Reducing adhesion affected veil advance by altering shaft adhesions. Moreover, the relationship between shaft adhesions and veil advance was robustly displayed during responses to natural guidance cues. Posterior sclerotome cells inhibited veil advance down the filopodia that contacted them and coordinately increased shaft adhesions. Schwann cells induced veil advance down contacting filopodia and coordinately abolished shaft adhesions. These observations support a model in which shaft adhesions control veil advance and in which axonal guidance cues control veil advance (and thereby alter the direction of growth cone travel) by modulating shaft adhesions (Fig. 9).

These results document a more precise and direct relationship between motile activities and adhesion than suspected previously and represent a substantial advance in our knowledge of how adhesive interactions modulate motility and guidance. Adhesions along individual filopodia had heretofore received little analysis, except by Smith (1994) who showed that filopodial tip adhesions stimulate filopodial engorgement only when shafts are nonadherent. Other studies have focused on less precise effects but are consistent with our model. Different adhesive environments affect the lamellar versus the filopodial morphology of cells and growth cones (Tosney and Landmesser, 1985; Theriot and Mitchison, 1991; Burden-Gulley et al., 1995; Lee and Jacobson, 1997; Aarts et al., 1998; Dogic et al., 1998), and experimentally altering adhesion alters overall growth cone form (Rivas et al., 1992; Varnum-Finney and Reichardt, 1994; for review, see Tanaka and Sabry, 1995; Are et al., 2001; Cox et al., 2001).

\section{Consequences for axonal guidance}

This study identifies a novel guidance mechanism in which contact at the filopodial tip reliably alters adhesion along the filopodial shaft and thereby alters veil advance and the direction of growth cone travel. One guidance cue tested here promotes shaft adhesions, whereas the other cue inhibits such adhesions. Because two different guidance signals both operate by altering shaft adhesion, this mechanism for controlling veil advance may be used widely. In addition to steering growth cones, this mechanism would potentiate responses to cues that are novel. The response would be most vigorous under conditions in which the shaft and tip contact two different substrates, as they would when the tip of a filopodium contacted a novel guidance cue. This mechanism is likely to operate in the embryo as well as in culture, because the distribution of the lamellar versus the filopodial form is clearly controlled by location-specific guidance signals (Tosney and Landmesser, 1985).

The relationship between adhesions and lateral stability also has implications for motility and environmental interactions. Filopodial merging may help filopodia mature into nascent neurites by supplying cytoplasmic elements that stimulate elongation and mediate thickening. Elongation is consistently stimulated by

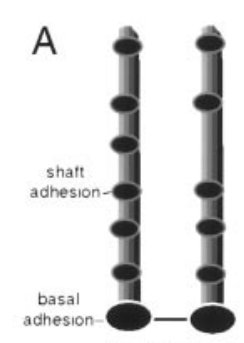

veil inhibited

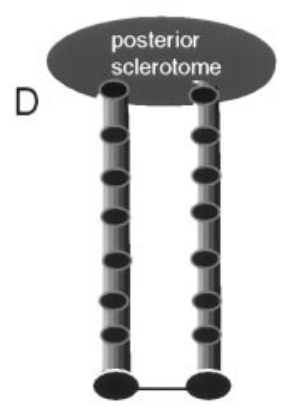

veil prohibited

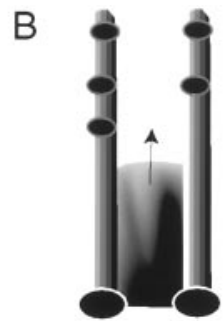

veil advances

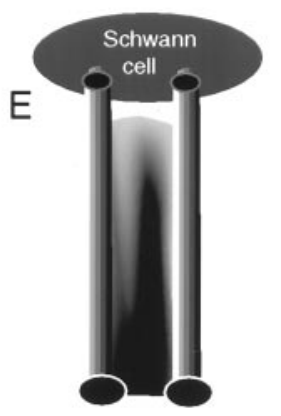

veil induced

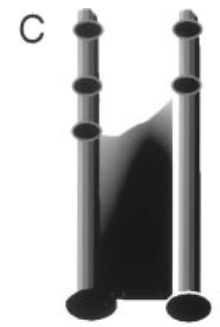

veil stops

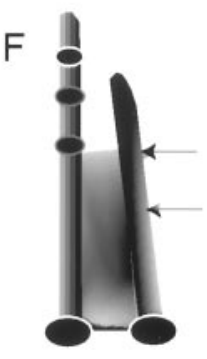

unstable
Figure 9. Model showing how shaft adhesions control veil (lamellar) advance. Note that all filopodia possess basal adhesions. $A-C$, Veil advance on laminin. $A$, Veils fail to advance down filopodia that have multiple shaft adhesions. $B$, Veils actively advance (arrow) down regions lacking shaft adhesions. $C$, Veils cease advance immediately proximal to a shaft adhesion. $D, E$, Guidance cues that alter veil advance alter filopodial adhesion patterns coordinately. $D$, When the tip of a filopodium contacts a posterior sclerotome cell, the guidance signal acts to promote adhesions between the filopodial shaft and laminin, thereby inhibiting veil advance. $E$, When the tip of a filopodium contacts a Schwann cell, the guidance signal acts to inhibit adhesions between the filopodial shaft and laminin, thereby promoting veil advance. $F$, Lateral instability. When two filopodia support a veil but one is nonadherent, then veils do not stabilize or retract; instead, the nonadherent filopodium and the veil move laterally (arrows) and merge with the adjacent, adherent filopodium.

merging. Similarly, filopodia elongate after merging in Aplysia growth cones (Oldenbourg et al., 2000). In sensory growth cones, multiple merging events produce thickened filopodia with abundant adhesions, consistent with early stages in neurite formation. Filopodial thickening and maturation were considered previously to result only from cytoplasmic engorgement (O'Connor et al., 1990; Myers and Bastiani, 1993; Steketee and Tosney, 1999), but merging is an alternative means of promoting filopodial maturation and growth cone advance, particularly on less adherent substrates.

\section{Surprising diversity in the function of substrate adhesions}

The idea that substrate adhesions within the same cell, much less within the same filopodium, can mediate distinct functions is novel. Previously, investigation of function focused on two easily detectable adhesion types: those at filopodial tips in neurons that mediate signal reception and "focal adhesions" in non-neuronal cells that regulate growth, differentiation, apoptosis, and motility (for review, see Tanaka and Sabry, 1995; Yamada, 1997). Focal adhesions were thought to be molecularly homogeneous (Geiger et al., 1995) until recently, when Zamir et al. (1999) documented extensive molecular diversity with, at the extremes, two major types distinguishable by morphology, regulation, and molecular profile. Possible molecular diversity in growth-cone adhesions previously could not be assigned functional significance because 
studies used static images (Letourneau and Shattuck, 1989; Arregui et al., 1994; Burden-Gulley and Lemmon, 1996; Renaudin et al., 1999). We were able to identify distinctive functions by directly correlating adhesion types with motile activities and responses to cues.

\section{Distinctive roles for tip, shaft, and basal adhesions}

In addition to mediating signaling, adhesions are thought to serve mechanical functions (for review, see Tanaka and Sabry, 1995). Adhesions can act as a "clutch" to restrict retrograde flow of f-actin filaments along the long axis of the filopodium (Lin and Forscher, 1995; Suter et al., 1998; Suter and Forscher, 2000) or as a tether to prevent lateral movement (current study). Clutch and tether activities likely interact. For example, the lateral movement of radial actin bundles in Aplysia neurons, mediated by retrograde flow (Oldenbourg et al., 2000), may be allowed because these filopodia lack adhesions. Mechanical actions are unlikely to be dependent on a particular signaling cascade. First, tethering at the tip is independent of which molecular "glue" maintains the adhesion. For instance, the duration of adhesion is similar regardless of whether tips adhere to laminin, Schwann cells, or posterior sclerotome (Steketee and Tosney, 1999; Polinsky et al., 2000). Second, process stability is disrupted after reducing vinculin (Varnum-Finney and Reichardt, 1994), a common component of all three filopodial adhesions. Third, all three types of adhesions similarly restrict lateral mobility regardless of their position or more distinctive functions, reflecting a common activity.

In growth cones, analysis of adhesions at filopodial tips provides the most convincing evidence that adhesions actively signal. Filopodial tips exhibit specializations in accord with roles in signaling, tethering, and f-actin dynamics (Wu and Goldberg, 1993; Mallavarapu and Mitchison, 1999). Most intriguing, adhesion at the tip alone can trigger signal cascades that alter cytoskeletal and motile activities (Dedhar, 1999; Isbister and O'Connor, 1999). For instance, tip adhesion to a cue can induce transient elevation of intracellular calcium within individual filopodia that promotes growth cone turning (Gomez et al., 2001). Tethering alone fails to account for specific responses that alter motility. The kind rather than the duration of tip adhesion mediates guidance responses (Bastmeyer and Stuermer, 1993; Oakley and Tosney, 1993; Isbister and O’Connor, 1999; Polinsky et al., 2000).

Shaft adhesions are clearly a target of environmental signaling. Their incidence is altered by guidance cues. Moreover, experiments directly establish that one guidance response is not attributable simply to tethering. When filopodia contact Schwann cells, antibodies to $\mathrm{N}$-cadherin block veil induction without releasing filopodial tips from the cell (Polinsky et al., 2000). The antibodies block signaling, not just tethering. Therefore, tip adhesion to Schwann cells must activate a signal that travels down the filopodium and rapidly prohibits laminin-based adhesions. Veil advance is thus subject to control of shaft adhesions by modulation of signaling cascades.

How the guidance signal alters shaft adhesion is unknown, but such alterations are not without precedent. In non-neuronal cells, adhesions based on one integrin isoform, $23 \beta 1$, can prohibit the formation of adhesions composed of another laminin isoform, a6 $\beta 1$ (Dogic et al., 1998). Several putative-signaling factors can antagonize integrin clustering or promote disassembly of focal adhesions, including elevated cAMP, growth factors, and altered levels of tyrosine phosphorylation (for review, see Dedhar and Hannigan, 1996; Angers-Loustau et al., 1999; Schoenwaelder and Burridge, 1999). Of most interest, a filopodial tip adhesion can stabilize the contacting filopodium and induce calcium transients in its shaft through integrin activation (Gomez et al., 2001), consistent with the possibility that these transients affect navigation by altering shaft adhesions.

What is most novel and exciting in the current study is the idea that a complex set of adhesion functions can be selectively displayed along the axis of single filopodia. In addition to a simple mechanical tethering to the substrate common to many adhesions, specific adhesions along a single filopodium play distinctive roles. These adhesions are identifiable by their position but also have structural, and likely molecular, differences. Tip adhesions suffice to initiate signals. Basal adhesions develop a distinctive structure, the focal ring, and stimulate signal cascades vital to actin filament organization and the emergence, orientation, and dynamics of filopodia (Steketee et al., 2001). They are long lived and label selectively for Rac1 (K. W. Tosney, unpublished observations). Shaft adhesions lack focal rings (Steketee et al., 2001), label selectively for Cdc42 (Tosney, unpublished observations), and control veil advance. Currently, the role and distribution of adhesions along actin bundles has received little attention in nonneuronal cells. In light of the robust relationship between shaft adhesions and veil advance, it will be important to determine whether similar adhesions control lamellar advance in other cells.

In conclusion, we show that veil advance is controlled by shaft adhesions in individual filopodia and that guidance cues can specifically alter veil dynamics by modulating signal cascades that alter shaft adhesions. We thus identify a guidance mechanism unsuspected previously. This study also augments evidence that adhesions arrayed along individual filopodia play distinct functional roles. These adhesions have important implications for axonal guidance mechanisms and for lamellar advance, both in growth cones and other cells, and further study of these adhesions is expected to reveal unique insights on how guidance signals control motility.

\section{REFERENCES}

Aarts L, Schrama WJ, Hage JL, Bos WH, Gispen H, Schotman P (1998) B-50/GAP-43-induced formation of filopodia depends on Rho-GTPase. Mol Biol Cell 9:1279-1292.

Angers-Loustau A, Cote J, Charest A, Dowbenko D, Spencer S, Lasky LA, Tremblay M (1999) Protein tyrosine phosphatase-PEST regulates focal adhesion disassembly, migration, and cytokinesis in fibroblasts. J Cell Biol 144:1019-1031.

Are A, Pinaev G, Burova E, Lindberg U (2001) Attachment of A-431 cells on immobilized antibodies to the EGF receptor promotes cell spreading and reorganization of the microfilament system. Cell Motil Cytoskeleton 48:24-36.

Arregui CO, Carbonetto S, McKerracher L (1994) Characterization of neural cell adhesion sites: point contacts are the sites of interaction between integrins and the cytoskeleton in PC12 cells. J Neurosci 14:6967-6977.

Bastmeyer M, Stuermer CA (1993) Behavior of fish retinal growth cones encountering chick caudal tectal membranes: a time-lapse study on growth cone collapse. J Neurobiol 24:37-50.

Bentley D, Toroian-Raymond A (1986) Disoriented pathfinding by pioneer neurone growth cones deprived of filopodia by cytochalasin treatment. Nature 323:712-715.

Bottenstein JE, Skaper SD, Varon SS, Sato GH (1980) Selective survival of neurons from chick embryo sensory ganglionic dissociates utilizing serum-free supplemented medium. Exp Cell Res 125:183-190.

Burden-Gulley SM, Lemmon V (1996) L1, N-cadherin, and laminin induce distinct distribution patterns of cytoskeletal elements in growth cones. Cell Motil Cytoskeleton 35:1-23.

Burden-Gulley SM, Payne HR, Lemmon V (1995) Growth cones are actively influenced by substrate-bound adhesion molecules. J Neurosci 15:4370-4381.

Burmeister DW, Goldberg DJ (1988) Micropruning: the mechanism of turning of Aplysia growth cones at substrate borders in vitro. J Neurosci 8:3151-3159.

Chien CB, Rosenthal DE, Harris WA, Holt CE (1993) Navigational errors made by growth cones without filopodia in the embryonic $X e$ nopus brain. Neuron 11:237-251. 
Cox EA, Sastry SK, Huttenlocher A (2001) Integrin-mediated adhesion regulates cell polarity and membrane protrusion through the Rho family of GTPases. Mol Biol Cell 12:265-277.

Dedhar S (1999) Integrins and signal transduction. Curr Opin Hematol 6:37-43.

Dedhar S, Hannigan GE (1996) Integrin cytoplasmic interactions and bidirectional transmembrane signaling. Curr Opin Cell Biol 8:657-669.

Desai CJ, Sun Q, Zinn K (1997) Tyrosine phosphorylation and axon guidance: of mice and flies. Curr Opin Neurobiol 7:70-74.

Dogic D, Rousselle P, Aumailley M (1998) Cell adhesion to laminin 1 or 5 induces isoform-specific clustering of integrins and other focal adhesion components. J Cell Sci 111:793-802.

Dogic D, Eckes B, Aumailley M (1999) Extracellular matrix, integrins and focal adhesions. Curr Top Pathol 93:75-85.

Fan J, Raper JA (1995) Localized collapsing cues can steer growth cones without inducing their full collapse. Neuron 14:263-274.

Gallo G, Letourneau PC (1999) Axon guidance: a balance of signals sets axons on the right track. Curr Biol 9:R490-R492.

Geiger B, Yehuda-Levenberg S, Bershadsky AD (1995) Molecular interactions in the submembrane plaque of cell-cell and cell-matrix adhesions. Acta Anat (Basel) 154:46-62.

Goldberg DJ, Burmeister DW (1986) Stages in axon formation: observations of growth of Aplysia axons in culture using video-enhanced contrast-differential interference contrast microscopy. J Cell Biol 103:1921-1931.

Goldberg DJ, Wu DY (1996) Tyrosine phosphorylation and protrusive structures of the growth cone. Perspect Dev Neurobiol 4:183-192.

Gomez TM, Robles E, Poo MM, Spitzer NC (2001) Filopodial calcium transients promote substrate-dependent growth cone turning. Science 291:1983-1987.

Hamburger V, Hamilton HL (1951) A series of normal stages in the development of the chick embryo. J Morphol 88:49-92.

Hammerback JA, Letourneau PC (1986) Neurite extension across regions of low cell-substratum adhesivity: implications for the guidepost hypothesis of axonal pathfinding. Dev Biol 117:655-662.

Isbister CM, O'Connor TP (1999) Filopodial adhesion does not predict growth cone steering events in vivo. J Neurosci 19:2589-2600.

Lee J, Jacobson K (1997) The composition and dynamics of cellsubstratum adhesions in locomoting fish keratocytes. J Cell Sci 110:2833-2844.

Letourneau PC (1975) Cell-to-substratum adhesion and guidance of axonal elongation. Dev Biol 44:92-101.

Letourneau PC, Shattuck TA (1989) Distribution and possible interactions of actin-associated proteins and cell adhesion molecules of nerve growth cones. Development 105:505-519.

Lin CH, Forscher P (1995) Growth cone advance is inversely proportional to retrograde f-actin flow. Neuron 14:763-771.

Mallavarapu A, Mitchison T (1999) Regulating actin cytoskeleton assembly at filopodial tips controls their extension and retraction. J Cell Biol 146:1097-1106.

Meiri K, Burdick D (1991) Nerve growth factor stimulation of GAP-43 phosphorylation in intact isolated growth cones. J Neurosci 11:31553164 .

Myers PZ, Bastiani MJ (1993) Growth cone dynamics during the migration of an identified commissural growth cone. J Neurosci 13:127-143.
Oakley RO, Tosney KW (1993) Contact mediated mechanisms of motor axon segmentation. J Neurosci 13:3773-3792.

O'Connor TP, Duerr JS, Bentley D (1990) Pioneer growth cone steering decisions mediated by single filopodial contacts in situ. J Neurosci 10:3935-3946.

Oldenbourg R, Katoh K, Danuser G (2000) Mechanism of lateral movement of filopodia and radial actin bundles across neuronal growth cones. Biophys J 78:1176-1182.

Polinsky M, Balazovich K, Tosney KW (2000) Identification of an invariant response: stable contact with Schwann cells induces veil extension in sensory growth cones. J Neurosci 20:1044-1055.

Renaudin A, Lehmann M, Girault JA, McKerracher L (1999) Organization of point contacts in neuronal growth cones. J Neurosci Res 55:458-471.

Rivas RJ, Burmeister DW, Goldberg DJ (1992) Rapid effects of laminin on the growth cone. Neuron 8:107-115.

Schoenwaelder SM, Burridge K (1999) Bidirectional signaling between the cytoskeleton and integrins. Curr Opin Cell Biol 11:274-286.

Smith CL (1994) Cytoskeletal movements and substrate interactions during initiation of neurite outgrowth by sympathetic neurons in vitro. J Neurosci 14:384-398.

Son YJ, Thompson WJ (1995) Schwann cell processes guide regeneration of peripheral axons. Neuron 14:125-132.

Steketee MB, Tosney KW (1999) Contact with isolated sclerotome cells steers sensory growth cones by altering distinct elements of extension. J Neurosci 19:3495-3506.

Steketee MB, Balazovich K, Tosney KW (2001) Filopodial initiation and a novel filament organizing center, the focal ring. Mol Biol Cell $12: 2378-2395$.

Suter DM, Forscher P (2000) Substrate-cytoskeletal coupling as a mechanism for the regulation of growth cone motility and guidance. J Neurobiol 44:97-113.

Suter DM, Errante LD, Belotserkovsky V, Forscher P (1998) The Ig superfamily cell adhesion molecule, apCAM, mediates growth cone steering by substrate-cytoskeletal coupling. J Cell Biol 141:227-240.

Tanaka E, Sabry J (1995) Making the connection: cytoskeletal rearrangements during growth cone guidance. Cell 83:171-176.

Tannahill D, Cook GMW, Keynes RJ (1997) Axon guidance and somites. Cell Tissue Res 291:275-283.

Theriot JA, Mitchison TJ (1991) Actin microfilament dynamics in locomoting cells. Nature 352:126-131.

Tosney KW, Landmesser LT (1985) Growth cone morphology and trajectory in the lumbosacral region of the chick embryo. J Neurosci 5:2345-2358.

Tsui H-CT, Schubert D, Klein WL (1988) Molecular basis of growth cone adhesion: anchoring of adheron-containing filaments at adhesive loci. J Cell Biol 106:2095-2108.

Varnum-Finney B, Reichardt LF (1994) Vinculin-deficient PC12 cell lines advance unstable lamellipodia and filopodia and have a reduced rate of neurite outgrowth. J Cell Biol 127:1071-1084.

Wu DY, Goldberg DJ (1993) Regulated tyrosine phosphorylation at the tips of growth cone filopodia. Cell Biol 123:653-664.

Yamada KM (1997) Integrin signaling. Matrix Biol 16:137-141.

Zamir E, Katz B-Z, Aota S-I, Yamada KM, Geiger B, Kam Z (1999) Molecular diversity of cell-matrix adhesions. J Cell Sci 112:1655-1669. 